\title{
New methods of forming and aligning the instrumental frames of absolute transit circle catalogs
}

\author{
E.R. Holdenried and T.J. Rafferty \\ United States Naval Observatory 3450 Massachusetts Ave. NW Washington DC, 20392-5420, U.S.A.
}

Received September 24, 1996; accepted January 31, 1997

\begin{abstract}
A new method of defining the declination axis of the instrumental frame of an absolute transit circle catalog has been developed utilizing observations from stars over the entire sky rather than, as in the past, observations of just circumpolar stars. This method allows the planetary observations to be used directly to align the axes of the instrumental frame thus constructed with DE200. The alignment process has been simplified and recast so that it does not feed back into the definition of the instrumental frame. A declination offset, which is unique to absolute transit circle observational catalogs, is discussed emphasizing the problem of its cross-correlation with rotation terms from the alignment process. When compared to "traditional" methods, these new procedures give similar results for those quantities in common but they also produce additional adjustments that were not accounted for previously.
\end{abstract}

Key words: astrometry — methods: data analysis — instrumentation: miscellaneous

\section{Introduction}

Transit circles can produce observations of the positions of celestial objects that are said to be absolute in the sense that the positions do not explicitly rely on any previous observations of these objects. The absolute positions are defined with respect to an internally consistent frame that is unique to the particular instrument. The uniqueness of this instrumental frame is a function, not only of the instrument itself, but also of the methods used to form the catalog. To form a compiled catalog of absolute observations from several instrumental catalogs these frames must be brought into coincidence. This is usually done by aligning the instrumental frames, by use of observations of solar system objects, to a dynamical one as defined by a standard ephemeris.

Send offprint requests to: E.R. Holdenried
It has been the practice at the U.S. Naval Observatory and other institutions involved in the production of compiled catalogs to align the instrumental frame of absolute transit circle catalogs with a dynamically defined system through corrections to the equinox point and equatorial plane, referred to as the equinox correction and equator correction, derived from observations of the Sun and major planets. The equinox and equator corrections would be solved for simultaneously with corrections to the orbital elements of each planet. The five to ten year duration of a transit circle program necessarily restricted this treatment to observations of the Sun, Jupiter and the planets interior to Jupiter. Only small fractions of the orbits of the outer planets could be covered during a typical program making unsuitable their inclusion in the solutions. With the release of JPL's DE200 ephemerides (Standish 1990), it was realized that the orbits of the planets contained therein, and therefore the dynamical system defined by them, were so well established that it was no longer necessary or desirable to use the observations from a single transit circle catalog to correct the orbital elements. The W1 $1_{\text {J00 }}$ (Holdenried, in press), an absolute catalog of star, Sun and planet positions observed by the Six-inch transit circle at the U.S. Naval Observatory in Washington DC during the years 1977-1982, is the first catalog in the series to follow this suggestion and eliminate from the alignment solutions the corrections to orbital elements.

This change in methodology offered an opportunity to reconsider on a more fundamental level the entire philosophy of the alignment process as well as the formation of the instrumental frame. As a result we have proposed a new approach. To explain this new approach and to give an appreciation for its divergence from the old the following historical digression is necessary.

\section{Discussion}

\subsection{Traditional method - equinox correction}

As was mentioned above, the alignment of the instrumental frame with a dynamical frame was accomplished 
through an adjustment to the equinox point and the equatorial plane. The equinox correction assumed that the poles of the two frames were coincident, and thus a simple rotation was all that was necessary to align the zero point of the right ascensions. As a result of the way in which the right ascensions were determined, the zero point of the instrumental system, before any rotation was applied, was very close to that of the catalog which supplied the data for the apparent places of a special set of stars known as clock stars. Usually this catalog was one of the series of Fundamentalkatalogs (FK) from the Astronomisches Rechen-Institut in Heidelberg.

\subsection{Traditional method - circumpolar solution}

Traditionally the equator correction was an adjustment to the declination system at the mean declination of the planetary observations and was used to determine the horizontal instrumental flexure $(F)$ and to develop corrections, $(\Delta \phi)$ and $(\Delta R)$, to, respectively, the assumed latitude and refraction constant. These three quantities $(\Delta \phi, \Delta R$, and $F)$ can be related through an expression of the form:

$$
\begin{aligned}
(\mathrm{O}-\mathrm{C})_{\delta}^{\prime}-(\mathrm{O}-\mathrm{C})_{\delta}= & 2 \Delta \phi+\Delta R\left(\tan z+\tan z^{\prime}\right)+ \\
& F\left(\sin z+\sin z^{\prime}\right)
\end{aligned}
$$

where:

$$
\begin{aligned}
(\mathrm{O}-\mathrm{C})_{\delta}^{\prime}= & \text { observed minus computed declination of a } \\
& \text { star at lower culmination } \\
(\mathrm{O}-\mathrm{C})_{\delta}= & \text { observed minus computed declination of the } \\
& \text { same star at upper culmination } \\
z^{\prime}= & \text { zenith distance of the lower culmination } \\
& \text { observation } \\
z= & \text { zenith distance of the upper culmination } \\
& \text { observation } \\
\Delta R= & \text { correction to the constant of refraction } \\
\Delta \phi= & \text { correction to the assumed latitude } \\
F= & \text { horizontal flexure. }
\end{aligned}
$$

Because this method utilizes lower culmination observations it requires stars that are circumpolar.

Since the mean epochs of the upper and lower culmination observations purposely were kept nearly the same, the computed positions canceled out and the solution was, therefore, considered absolute. However, a solution including both the flexure $(F)$ and the correction to the latitude $(\Delta \phi)$ was not feasible because, over the small range of zenith distances involved, $F$ and $\Delta \phi$ are strongly correlated. To alleviate this problem, the value of the flexure empirically determined using two horizontal collimating telescopes was substituted into the equation. Typically the resulting flexure showed a very large scatter (for the $\mathrm{W}_{\mathrm{J} 00}$ program, the flexures had a standard deviation of \pm "'252). Though the flexure measured employing the collimators was poorly determined, it was necessary to use it because of the impossibility, when just the circumpolar stars were included in the solution, of solving simultaneously for the flexure and the corrections to the constant of refraction and the assumed latitude. Various theories have been proposed to explain why the flexure determined from the collimators is so poorly determined; probably the most reasonable postulates that temperature gradients in the air in the tube of the instrument introduce refraction effects that distort the measures. A study based on Washington, Six-inch data, by Høg \& Miller (1986), showed that flexures measured using the collimators varied with the rate of change of temperature leading to the conclusion that internal tube refraction was responsible for this behavior. A follow-up study, by $\mathrm{H} ø$ g \& Fabricius (1988) involving the Carlsberg transit circle, reinforced the conclusions about internal refraction and suggested the use of fans to mix the air. The construction of the Six-inch (specifically the exposed wires in the micrometer), however, precluded the adoption of that suggestion. During the $\mathrm{W} 1_{\text {Joo }}$ program the application of flexure as a function of the rate of change of temperature was tested; but very little, if any, improvement could be detected (see Table 1, line 2).

In any case, by substituting the mean flexure value measured using the collimators for $F$ in Eq. (1), $\Delta \phi$ and $\Delta R$ can be determined. Then by using the following:

$\Delta \delta=\Delta \phi+\Delta R \tan z+F \sin z$,

all the declinations were adjusted by the amount $\Delta \delta$. However, when this procedure was applied to the planets, it frequently resulted in a sizeable systematic offset in the $(\mathrm{O}-\mathrm{C})_{\delta}$ 's, traditionally called the equator correction. In the case of the $\mathrm{W} 5_{50}$ catalog (Hughes 1982) this offset was -0 ". 347 . Since the instrumental pole was regarded as absolutely fixed, an additional step was taken in which a solution was made for further corrections to $\Delta \phi$ and $\Delta R$, as well as a correction to $F$, by defining a model that smoothly distributed the offset between the pole and the equator so that positions near the pole were unaffected while the full offset was applied to those near the equator. This would seem to have the effect of producing a system whose pole was that of the instrument but with an equator that was not necessarily connected to that pole and with intermediate declinations that were only partially linked to either the pole or the equator. This method was employed for the $\mathrm{W}_{50}$ (Hughes 1982), WL 50 (Hughes 1992) and other Washington absolute catalogs, and, because of the location of the stars involved, will be referred to as the circumpolar solution.

The circumpolar solution is very weakly defined because of the sparseness of the set of stars contributing 
Table 1. Various solutions for the horizontal instrumental flexure $(F)$, the correction to the refraction constant $(\Delta R)$, and the correction to the assumed latitude $(\Delta \phi)$. Solution 1 simultaneously solves for $F, \Delta R$, and $\Delta \phi$ using only the circumpolar stars. This solution is very poor due to the strong correlation between $F$ and $\Delta \phi$ over the small range of the zenith distances of the circumpolar stars. Solution 2 applies values of the horizontal flexure, measured by means of collimators as a function of the rate of change of temperature, to the observations and subsequently solves for just $\Delta R$ and $\Delta \phi$ using only the circumpolar stars. Solution 3 applies the mean flexure value determined from the collimators to the observations and subsequently solves for just $\Delta R$ and $\Delta \phi$ using only the circumpolar stars. Solution 4 simultaneously solves for $F, \Delta R$, and $\Delta \phi$ using observations distributed over the entire sky and averaged into zones 2.5 degrees wide arranged sysmetrically about the zenith. When the data is arranged in this manner the solution for the coefficents, $F$ and $\Delta R$, is independent of the intercept, $\Delta \phi$, because of the properties of odd, trigonometric functions. In solution 5 the data is handled in practically the same fashion as for solution 4 , the only difference being that, initially, only $F$ and $\Delta R$ are solved for (line 5a). These values are then applied and $\Delta \phi$ determined (line 5b). The values for $F$ and $\Delta R$ are identical on lines 4 and 5 a because of the just mentioned behavior of odd, trigonometric functions. Solutions $5 \mathrm{a}$ and $5 \mathrm{~b}$ used together are referred to as the combined all-sky solution

\begin{tabular}{|c|c|c|c|c|c|}
\hline \multirow[b]{2}{*}{ no. } & \multirow[b]{2}{*}{ solutions } & \multirow{2}{*}{$\begin{array}{l}\text { Empirical Values } \\
F\left(^{(\prime)}\right)\end{array}$} & \multicolumn{3}{|c|}{ Calculated Values } \\
\hline & & & $F\left(^{\prime \prime}\right)$ & $\Delta R\left({ }^{\prime \prime}\right)$ & $\Delta \phi\left({ }^{\prime \prime}\right)$ \\
\hline 1 & Circumpolar & & $-1.629 \pm .524$ & $-0.081 \pm .084$ & $+1.637 \pm .161$ \\
\hline 2 & Circumpolar & $F(\Delta t)$ & & $+0.039 \quad .017$ & $\begin{array}{ll}-0.228 & .031\end{array}$ \\
\hline 3 & Circumpolar & $+0.662 \pm .252$ & & $+0.055 \quad .017$ & $\begin{array}{ll}-0.292 \quad .031\end{array}$ \\
\hline 4 & All-sky & & $+0.687 \quad .083$ & $\begin{array}{ll}-0.024 \quad .022\end{array}$ & $\begin{array}{ll}-0.186 \quad .007\end{array}$ \\
\hline$\overline{5 a}$ & Combined All-Sky (all-sky) & & +0.687 .158 & $-0.024 \quad .085$ & \\
\hline $5 b$ & Combined All-Sky (circumpolar) & & & & $-0.172 \quad .009$ \\
\hline
\end{tabular}

$(\mathrm{O}-\mathrm{C})_{\delta}$ 's and their limited range of zenith distances. Since the objective is to produce an instrumental system that is free of bias, such behavior is a source of concern. For example, if a breakdown in the solution produces an error in $\Delta R$, this error will manifest itself as a bias in the system that has a signature of the tangent of the zenith distance. Also, because of the aforementioned correlation between the $F$ and $\Delta \phi$, the circumpolar solution requires an initial value for the flexure. Although, in principle, the flexure can be measured using the collimators, it is evident from long experience that this flexure cannot be applied in a simple manner to other pointings of the instrument at the level of accuracy required. This is underscored by the fact that in past Washington transit circle catalogs the final adopted flexure differed significantly from the empirical. In the case of the $\mathrm{W} 5_{50}$, the measured flexure was 0 '.012 while the adopted value derived from the circumpolar solution was $0^{\prime \prime} 237$.

\subsection{New method - all-sky solution}

For the reasons enumerated above, we felt that a new approach would be of benefit in the discussion of the future catalogs. It was judged that $F$ and $\Delta R$ could be determined more accurately from star observations that were not restricted to just those that were circumpolar. Using the equation (for upper culmination observations)

$(\mathrm{O}-\mathrm{C})_{\delta}=\Delta \phi+\Delta R \tan z+F \sin z$

and (for lower culmination observations)

$(\mathrm{C}-\mathrm{O})_{\delta}^{\prime}=\Delta \phi+\Delta R \tan z+F \sin z$ for the stars observed at all zenith distances, all three unknowns $(\Delta \phi, \Delta R$, and $F)$ can be solved for by least squares. Because zenith distances are not restricted as they are in the circumpolar solution, the strong correlation between $\Delta \phi$ and $F$ is broken. However, the explicit introduction of $\mathrm{C}$, the star's computed place, will threaten the absolute quality of a catalog. It can be said though, in general, that the systematic errors in the C's are not correlated with the zenith distance or, more precisely, with the sine and tangent of the zenith distance. Furthermore, by grouping the $(\mathrm{O}-\mathrm{C})_{\delta}$ 's into zones arranged symmetrically about the zenith, we can take advantage of a useful feature of odd, trigonometric functions (such as sine and tangent). A least squares solution for the coefficients of such functions have the property of being completely independent of the intercept as long as the data points are distributed absolutely symmetrically about the origin. Thus a solution for $\Delta R$ and $F$, from data properly arranged, need not be dependent on $\Delta \phi$. This fact can be seen in Table 1 , lines 4 and $5 \mathrm{a}$; whether $\Delta \phi$ is included in the solution does not affect the values of $\Delta R$ and $F$. However, systematic errors in the C's will impact directly on $\Delta \phi$ in a cumulative fashion unless steps are taken to mitigate them. To accomplish this we have solved for $\Delta \phi$ separately using only circumpolar observations. By restricting the observations in this way, the systematic errors in the C's should practically (to the extent that the mean epochs of the upper and lower culmination observations are the same) cancel out. We shall refer to these methods, collectively, (i.e. both solving for all three terms simultaneously 
and solving for $F$ and $\Delta R$ separately from $\Delta \phi$ ) as the all-sky solution.

To summarize, the values of $F$ and $\Delta R$ from the allsky solution can be considered independent of the C's, but this is not the case for $\Delta \phi$. The value of $\Delta \phi$ necessarily will link the instrumental system to the system of the C's. As a regression problem, the values for $F$ and $\Delta R$ can be obtained, without including $\Delta \phi$, from the all-sky method using the model:

$(\mathrm{O}-\mathrm{C})_{\delta}^{*}=\Delta R \tan z+F \sin z$

where $(\mathrm{O}-\mathrm{C})_{\delta}^{*}=+(\mathrm{O}-\mathrm{C})_{\delta}$ for upper culmination observations and for lower culmination $(\mathrm{O}-\mathrm{C})_{\delta}^{*}=$ $-(\mathrm{O}-\mathrm{C})_{\delta}^{\prime}$. These values may then be applied to the observations and a solution for $\Delta \phi$ may be determined from the circumpolar stars using:

$(\mathrm{O}-\mathrm{C})_{\delta}^{\prime}-(\mathrm{O}-\mathrm{C})_{\delta}=2 \Delta \phi$

By combining the all-sky and circumpolar solutions, the absolute nature of the instrumental system is retained. We shall refer to this method as the combined all-sky solution.

In the discussion of the W1 $1_{\text {J00 }}$, only the FK5, Part I (Fricke 1988) stars were used in the combined all-sky solution. Comparisons were made between the circumpolar (Table 1, lines 2-3), the all-sky (Table 1, line 4), and the combined all-sky solutions (Table 1 , lines $5 \mathrm{a}$ and $5 \mathrm{~b}$ ). For the $\mathrm{W} 1_{\text {Jo0 }}$, the value of the flexure produced by the allsky solution is nearly identical to the value measured with respect to the collimators. This is probably coincidence for we have not seen this happen in other Washington catalogs. The major difference between the two methods is in the value for $\Delta R$. Since the correction to the constant of refraction is a function of $\tan z$, even small differences in $\Delta R$ will have a large effect at the extreme zenith distances. To the north the effect is not significant because lower culmination observations, which have the largest zenith distances, are not usually reported in the final catalog; but to the south the effect can be much more of a problem. To ascertain which method results in the "best" $\Delta R$, comparisons were made with positions of FK5 stars observed by the Carlsberg transit circle between 1986 and 1990 (Carlsberg 1989, 1991 and 1992) located at La Palma in the Canary Islands. This transit circle is at a latitude $20^{\circ}$ south of that of the instrument that observed the $\mathrm{W} 1_{\mathrm{J} 00}$, which allows the Carlsberg instrument to observe at moderate zenith distances the same stars that are very near the southern horizon of the Washington instrument. Thus the Carlsberg observations, for these stars, are considerably less impacted by refraction. When comparing the positions for the southern-most stars, those adjusted by the values from the all-sky solution agreed more closely with the Carlsberg results than did the positions adjusted by values from the circumpolar solution. A constant was applied to the Carlsberg observations so that the mean $(\mathrm{O}-\mathrm{C})_{\delta}$ 's were the same for the two instruments thus eliminating the effect of $\Delta \phi$. It is interesting that the $\Delta \phi$ 's differ significantly between the all-sky and the circumpolar solutions even though in each case they seem well determined. This is probably a result of the differing values for $\Delta R$.

\subsection{Instrumental frame alignment}

An important feature of the combined all-sky solution is that it does not utilize observations of solar system objects, thus leaving them free to be used to align the axes of the instrumental frame with the dynamical reference system. We will posit that the instrumental coordinate frame is a rigid system of orthogonal axes, and that the alignment with the dynamical frame should be accomplished by simple orthogonal transformations. If a rectangular coordinate system is employed the transformation can be accomplished by three rotations, one about each of the the axes. We will choose the axes in the usually manner so that the $z$ axis is parallel to the celestial pole, the $x$ axis points to the Vernal Equinox, and rotations are defined as positive in the right-handed sense. A rotation about the $x$ axis is described by the angle $i$, about the $y$ axis by the angle $j$ and about the $z$ axis by the angle $k$. To make the problem amenable to solution by linear least squares it is customary to utilize the small angle approximations of; $\sin a=a, \cos a=1$, and $\cos a \cos b=1$ (where $a$ and $b$ are small angles). In this way the rotation matrix reduces to

$[\mathbf{R}]=\left[\begin{array}{ccc}1 & -k & j \\ k & 1 & -i \\ -j & i & 1\end{array}\right]$

The equations of condition can be formulated as

$[\mathbf{R}]\left[\begin{array}{l}x \\ y \\ z\end{array}\right]=\left[\begin{array}{l}x^{\prime} \\ y^{\prime} \\ z^{\prime}\end{array}\right]$

where:

$x=\cos \delta \cos \alpha \quad(\alpha=$ right ascension, $\delta=$ declination $)$

$y=\cos \delta \sin \alpha$

$z=\sin \delta$

and the primed variables are the rectangular coordinates after being rotated. This procedure has become so common in problems in astrometry involving coordinate transformations that it has been called the standard method (Vityazev 1994), a term that we shall also use.

We tested the ability of the standard method to determine the rotational angles in the transformation between two frames and also to apply the rotations. This was done by running numerical experiments with data from a synthetic, observed catalog in which random errors could be injected that were typical of a transit circle along with 
known rotations between the catalog axes and the dynamical frame. Two types of data sets were treated; planetary data, which were restricted to "observations" located near the ecliptic, and star data, which included "observations" at all declinations. The standard method proved to be accurate (in the sense of mean residual) to better than $\pm 0^{\prime \prime} 002$ over the range of expected rotation angles $\left(0^{\prime \prime}-10^{\prime \prime}\right)$.

The angles resulting from the solution by the standard method using 2062 planetary observations (Sun, Mercury, Venus and Mars) from the W1 $1_{J 00}$ and the ephemeris from DE200 are:

$$
\begin{aligned}
i & =+0 . .097 \pm \text { ".031 } \\
j & =+0 . .044 \pm \text { ".029 } \\
k & =-0.223 \pm \text { ".023. }
\end{aligned}
$$

For the purposes of comparison, the same set of data was treated by what may be described as the "traditional" method for aligning an instrumental transit circle frame with the dynamical. That is, a least squares solution for an equator and equinox correction was performed employing the same programs that in the past were used to solve for corrections to the orbital elements. Forcing the corrections to the orbital elements to be zero resulted in an equinox correction of $-00^{\prime \prime} 225 \pm$.'008. As expected this value is very close to $k$, a rotation about the $z$-axis, since the equinox correction represents a rotation about the celestial pole, which is parallel to the $z$-axis. The angles $i$ and $j$, representing an inclination of the celestial pole, have no analogs in the traditional solution.

\subsection{Declination offset}

After the rotations determined by the standard method have been applied to the planet observations, a significant offset in declination (i.e. the mean of the planetary $(\mathrm{O}-\mathrm{C})_{\delta}$ 's) frequently remains. It could be argued that, to align the transit circle's instrumental system to the dynamical system, this declination offset must be accounted for in some fashion. However to do so, two problems must be solved.

First, as others have demonstrated convincingly (Vityazev 1994), non-rotational differences between coordinate systems can be cross-correlated with the rotational ones making them very difficult to separate. The declination offset is clearly a non-rotational term and as such may confound the determination of rotation angles. Vityazev has shown that non-rotational terms should be removed before a solution for rotational terms can be attempted. Unfortunately, in the problem we are addressing here, there is no a priori source of information that would allow us to remove the declination offset from the data. However, it may be possible to carefully choose the data in such a way as to break the correlation between the offset and the rotation angles. That this might be so, is intuitively evident in the case of "perfect" data. Perfect data would be distributed evenly in right ascension and declination. With this data the determination of rotation angles is completely uncorrelated with a declination offset.

Our study has shown that if one chooses to solve for the rotation angles and offset simultaneously by least squares using the equations of condition

$$
\begin{aligned}
& (\mathrm{O}-\mathrm{C})_{\delta}=-i \sin \delta+j \cos \alpha+\lambda \\
& (\mathrm{O}-\mathrm{C})_{\alpha}=+i \tan \delta \cos \alpha+j \tan \delta \sin \alpha-k
\end{aligned}
$$

where $\lambda=$ declination offset

that cross correlations of greater than $40 \%$ (from the correlation matrix) indicate unstable solutions and poorly distributed data. It is the distribution of the observations in right ascension that is most important. We have found if cross-correlations are greater than $40 \%$, then that data set cannot be used to solve for rotation angles unless the declination offset can be estimated and removed beforehand.

The second problem involving the declination offset is demonstrated in Tables 2-4. These tables summarize solutions, by the standard method, for rotations (between the instrumental frame and DE200), and after the rotations have been applied, a determination of $\lambda$ for data sets comprised of various combinations of solar system observations (the Sun and major planets) from the three absolute catalogs of transit circles observations, W1J00, Capetown observations between 1951 and 1959 (Stoy 1968), and Greenwich observations between 1942 and 1954 (Tucker 1983). Each line in the tables gives the angles of rotation $(i, j, k)$ as well as $\lambda$ produced by treating the $(\mathrm{O}-\mathrm{C})$ 's of different combinations of solar system objects. Also, the columns labeled "traditional" give the results obtained by code that solved for equator and equinox corrections along with corrections to the orbital elements, but, here for comparison purposes only, the orbital elements were forced to zero. As the tables show, the angle $k$ is equivalent to the traditional equinox correction and the $\lambda$ is equivalent to the traditional equator correction. The tables are divided into two parts, the upper part contains data sets that were judged to have acceptable distributions; acceptable in the sense, as explained previously, of being suitable for solutions for rotations that are not correlated with $\lambda$. The results shown in the lower part of the table and marked by asterisks were judged, by this criteria, not to be acceptable. Note that $\lambda$ agrees most closely with the traditional equator correction for the acceptable data. It is perhaps more important to note, however, that even among the acceptable data sets $\lambda$ varies significantly. All three catalogs suffer from this behavior.

Such behavior might be explained by problems with DE200, but we think this is unlikely since the three catalogs show variations in $\lambda$ that are not consistent between the instruments. We think it more probable that the observations themselves are the cause. For transit circle 
Table 2. Summary of the solutions by the standard method for the rotation angles $(k, j$, and $i)$ between the axes of the instrumental reference frame of a W1J00 Catalog (1977-1982) and DE200 for various combinations of planets. A declination offset is also solved for. The columns labeled "traditional" give results obtained from solutions for the equator and equinox corrections along with the corrections to the orbital elements, with the corrections to the orbital elements forced to zero. The planet groups marked with an asterisk are poorly distributed in this catalog, and these solutions for the rotation angles and declination offset are weak

W1J00 (1977-1982)

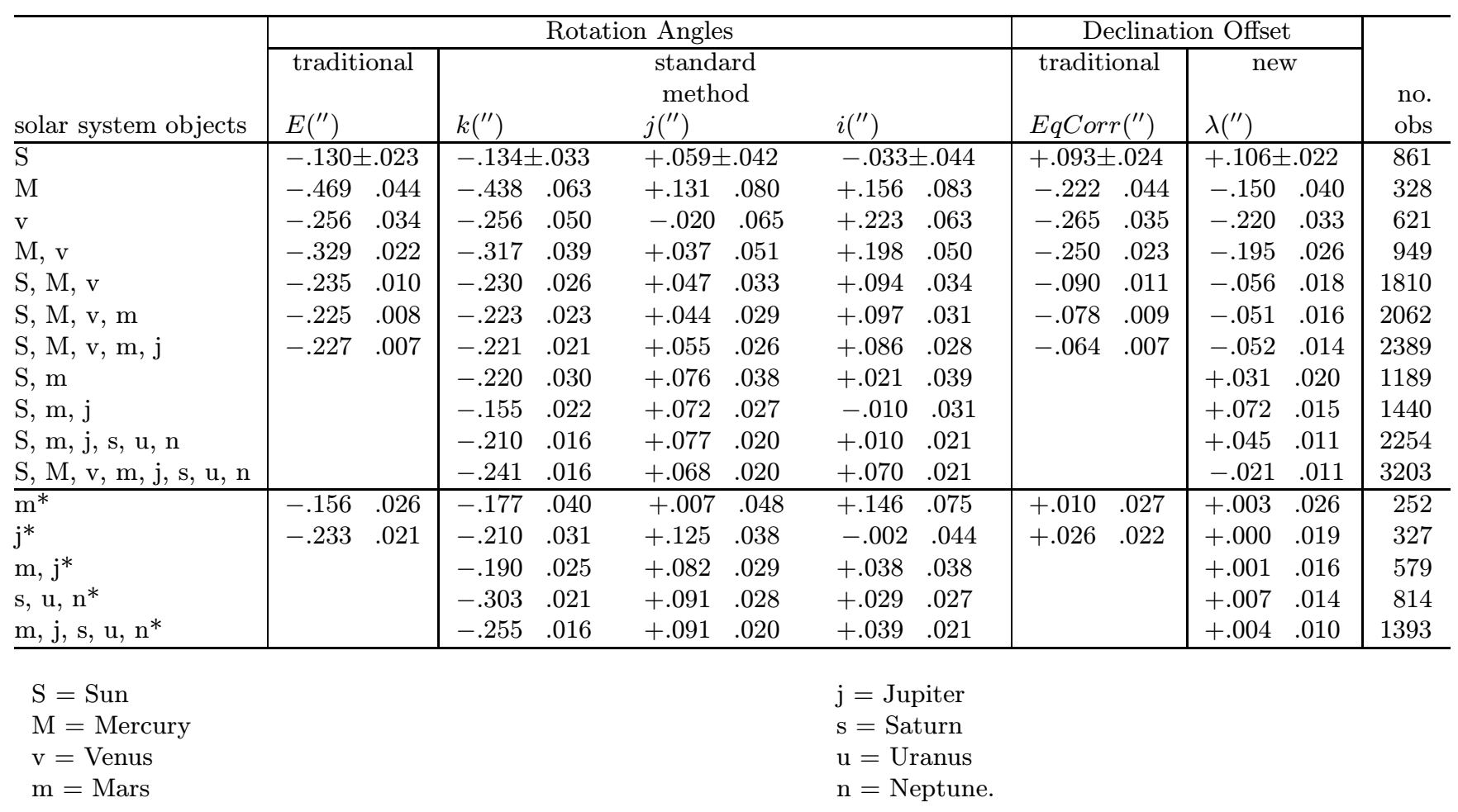

observations, a non-zero $\lambda$ may be introduced through an erroneous correction to the assumed latitude. However, in the case of the $\mathrm{W} 1_{\mathrm{J} 00}$, the correction to the assumed latitude is very well determined either from the all-sky solution (solving for the flexure, correction to the constant of refraction, and the correction to the assumed latitude, Table 1, lines 4 and 5b) or the circumpolar solution (solving for just the correction to the assumed latitude, Table 1 , lines 2 and 3 ). Another source of uncertainty is the effect of making observations in two very different environments, daytime and nighttime. The Sun, Mercury, and Venus can only be observed in the daytime while the remainder of the solar system objects are usually observed only at night. Systematic differences between the daytime and nighttime observations are known to exist and are corrected for by an analysis of the observations of the same stars observed during the day and the night. In the case of the $\mathrm{W} 1_{\mathrm{J} 00}$, this analysis produced night-minus-day corrections as function of hour angle of the Sun, declination, and temperature. A further source of uncertainty is the method of observing the solar system objects. For Mercury, the "center of light" is observed with corrections applied for the phase. For Venus, the two illuminated limbs are observed and corrections applied to reduce the observations to the center of the planet. For the $\mathrm{W} 1_{\mathrm{J} 00}$, the $\mathrm{W} 5_{50}$, and other Washington catalogs, additional corrections to both Mercury and Venus due to phase were determined empirically and also applied. Tables $2-4$ show the rotation angles and $\lambda$ values determined from Mercury and Venus to have the greatest differences from among the various solar system objects. Unfortunately DE200 has been constructed in such a way as to exacerbate these problems. That is, the solar system objects given the highest weight in defining the dynamical frame are daytime and/or extended objects, these are the Sun (i.e the reflex of the Earth's motion), and (because of radar ranging and spacecraft fly-bys) Mercury, Venus, and Mars. For the W1 $1_{J 00}$, it is surprising how consistent the results are for the outer planets. However for these planets there is evidence of systematic errors in DE200 which can be as large as \pm 0 "' 2 , 
Table 3. Summary of the solutions by the standard method for the rotation angles $(k, j$, and $i)$ between the axes of the instrumental reference frame of a Greenwich Catalog (1942-1954) and DE200 for various combinations of planets. A declination offset is also solved for. The columns labeled "traditional" give results obtained from solutions for the equator and equinox corrections along with the corrections to the orbital elements, with the corrections to the orbital elements forced to zero. The planet groups marked with an asterisk are poorly distributed in this catalog, and these solutions for the rotation angles and declination offset are weak

Greenwich (1942-1954)

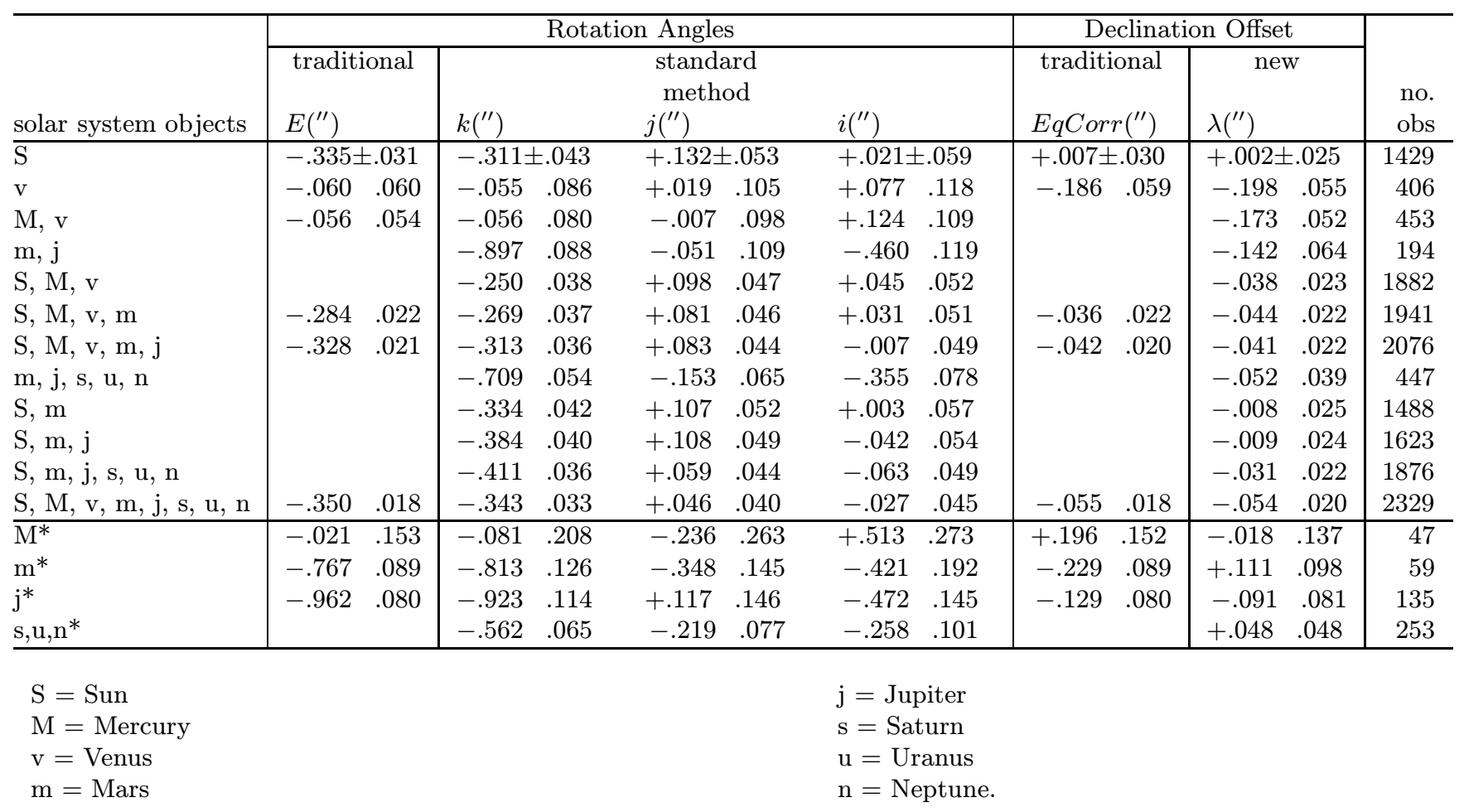

depending on date. It might be possible to utilize observations of outer planets if an ephemeris was developed based on more recent data than was included in DE200, and if that ephemeris was adopted as the dynamical standard. Currently, though, it is the general consensus, that the combination of planets interior to Jupiter represent the best realization of the coordinate frame of DE200. The solar system objects that would be least effected by any of the above described problems are the minor planets. Lamentably, although 5 minor planets are used in the perturbation models, there are no ephemerides for them included in DE200 or any of its current successors.

If we had more confidence in the planetary observations from the transit circle, we would apply $\lambda$ to complete the alignment of the instrumental system to the dynamical system. At this time we hesitate to do this because of the problems discussed above and because the value of the offset varies so much between the individual and combined groups of solar system objects. The Pole-to-pole program (Corbin 1988), for which the observations were recently completed (1985-1996), using transit circles in Washington and New Zealand might resolve the problems with the daytime observations. The Washington transit circle made visual observations whereas the New Zealand transit circle used an image dissector, thus the reductions of observations of extended objects such as Mercury and Venus will differ considerably between the two instruments. The New Zealand transit circle, also, was able to observe a great many more daytime stars and should be able to improve the determination of the night-minus-day correction (Rafferty \& Loader 1995). Both transit circles observed Mars during the daytime and nighttime, which also should improve the determination of the night-minus-day correction. The observations of each instrument can be reduced using the methods introduced to the $\mathrm{W} 1_{\text {J00 }}$ and comparisons made to see if applying $\lambda$ improves the agreement between the two programs. Finally there are efforts under way by others to include minor planets in the dynamical frame. 
Table 4. Summary of the solutions by the standard method for the rotation angles $(k, j$, and $i)$ between the axes of the instrumental reference frame of a Capetown Catalog (1951-1959) and DE200 for various combinations of planets. A declination offset is also solved for. The columns labeled "traditional" give results obtained from code which produced solutions for the equator and equinox corrections along with the corrections to the orbital elements, with, for the purposes of this comparison, the corrections to the orbital elements forced to zero. The planet groups marked with an asterisk are poorly distributed in this catalog, and the solutions for the rotation angles and declination offset are weak

Capetown (1951-1959)

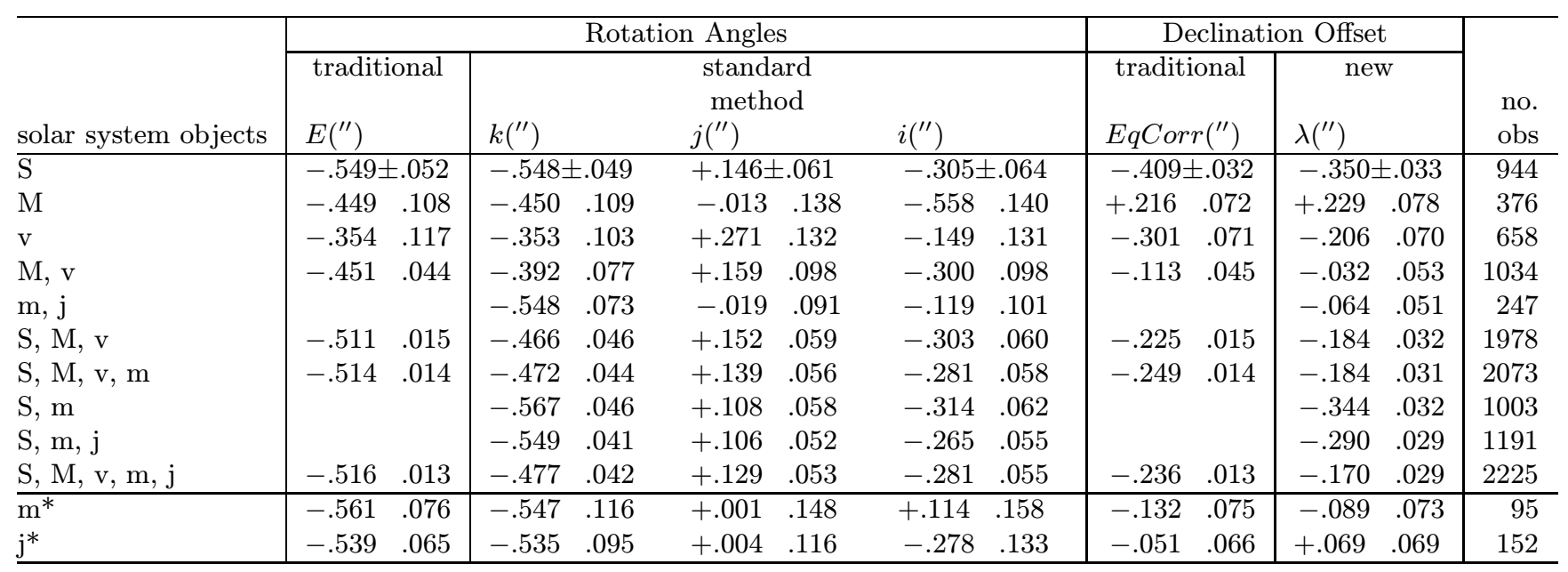

$$
\begin{aligned}
& \mathrm{S}=\text { Sun } \\
& \mathrm{M}=\text { Mercury } \\
& \mathrm{v}=\text { Venus. }
\end{aligned}
$$

$\mathrm{m}=$ Mars

$\mathrm{j}=$ Jupiter
It has been pointed out that, in the near future, the IAU will adopt the VLBI positions of some 600 extragalactic radio sources as the definition of the new International Celestial Reference Frame (ICRF) (Morrison 1997). The optical counterpart of this frame will be realized by the Hipparcos Catalogue, which promises to be co-aligned with the ICRF to better than 0.6 mas at epoch J1991.25. Of course, the standard method of alignment can just as easily be used to rotate transit circle catalogs into this system (i.e. the ICRF as represented by the Hipparcos Catalog) as it was used to rotate them into a dynamical system. Indeed, such a scheme offers the possibility of determining the rotational offsets between the dynamical reference system the ICRF. However, because of the degradation of the Hipparcos Catalog over time caused by proper motions, the treatment of transit circle catalogs at epochs more that $30-40$ years from the mean epoch of Hipparcos (1991.25) will require the use of some other system. At present the best system for covering the widest range in epochs is DE200. It is planned that the Washington Fundamental Catalog (Corbin 1995), which will include absolute transit circle catalogs from the early 1900 's, will make use of the standard method to align all the catalogs to a common system, probably DE200 or a more current version of it. It should then be possible, using the same algorithm, to rotate everything into the system of Hipparcos.

\section{Conclusion}

In forming the declination part of the instrumental frame of an absolute transit circle catalog, the combined allsky solution is superior to the traditional method that only utilized observations of circumpolar stars. Indeed, we have shown that the circumpolar solutions may have introduced systematic biases into the catalogs. Further, we have shown that, if the combined all-sky solution is adopted, the instrumental frame may be aligned to a dynamical one by simple rotations about the axes of a rectangular coordinate system. The problems of the declination offset may be handled if care is taken in choosing a well distributed set of planetary observations from which the rotation angles necessary for alignment are derived. The Pole-to-pole program holds the promise of providing new information on this problem and the problems presented by the visual appearance of extended objects. Solutions involving three transit circle catalogs demonstrate that the declination offset is similar to the "traditional" equator correction and that the standard method gives an 
adjustment equivalent to the traditional equinox correction, as well as additional adjustments that were not accounted for by the older method.

Acknowledgements. We wish to thank Drs. T.E. Corbin and G.H. Kaplan for their many helpful discussions and in particular for the suggestion of treating the alignment process as a strict rotation problem. We would like to express our appreciation to Z.G. Yao, formerly of Sachs Freeman Associates and working at the U.S. Naval Observatory, for the data in Tables 2-4 in the columns labeled "traditional". We would also like to recognize our referee for his many helpful comments and suggestions, and particularly for the much valued discussions on organization and clarity. And finally we would like to thank Dr. M. Germain for his insights into the properties of least square solutions of trigonometric functions.

\section{References}

Carlsberg Meridian Catalogue La Palma No. 4. Observations of positions of stars and planets: May 1984 to February 1988. (CMC4): 1989, Copenhagen University Observatory, Royal Greenwich Observatory and Real Instituto y Observatorio de la Armada en Su Fernando

Carlsberg Meridian Catalogue La Palma No. 5. Observations of positions of stars and planets: May 1988 to December 1989. (CMC5): 1991, Copenhagen University Observatory, Royal Greenwich Observatory and Real Instituto y Observatorio de la Armada en Su Fernando

Carlsberg Meridian Catalogue La Palma No. 6. Observations of positions of stars and planets for the year 1990. (CMC6): 1992, Copenhagen University Observatory, Royal Greenwich Observatory and Real Instituto y Observatorio de la Armada en Su Fernando

Corbin T.E., 1995, Large Fundamental and Global Transit Circle Catalogs, IAU Symposium No. 166, Astronomical and Astrophysical Objectives of Sub-milliarcsecond Optical Astrometry. In: Høg E. and Seidelmann P.K. (eds.). Kluwer Academic Publishers, pp. 45-48
Corbin T.E., 1988, McNally D. (ed.) Transactions of the IAU (Vol. XXB), p. 132

Fricke W., Schwan H., Lederle T., 1988, Fifth Fundamental Catalog, Part I. The Basic Fundamental Stars, Astronomisches Rechen-Institut, Heidelberg

Høg E., Miller R.J., 1986, Internal Refraction in a USNO Meridian Circle, AJ 92, 495-502

Høg E., Fabricius C., 1988, Atmospheric and Internal Refraction in Meridian Observations, A\&AS 196, 301-312

Holdenried E.R., Rafferty T.J., Results of observations made with the Six-inch transit circle 1977-1982 (W1 Joo), Publications of the United States Naval Observatory, Second Series (in press)

Hughes J.A., Scott D.K., 1982, Results of observations made with the Six-inch transit circle 1963-1971 (W550), Publications of the United States Naval Observatory, Second Series, XXIII(3)

Hughes J.A., Smith C.A., Branham R.L., 1992, Results of observations made with the Seven-inch transit circle 1967$1973\left(\mathrm{WL}_{50}\right)$, Publications of the United States Naval Observatory, Second Series, XXVI(2)

Morrison L.V., 1997 (private communication)

Rafferty T.J., Loader B.R., 1993, Improvements in the use of daytime observations from a transit circle, A\&AS 271, 727733

Standish E.M. Jr., 1990, The Observational Basis for JPL's DE200, the planetary Ephemerides of the Astronomical Almanac, A\&AS 233, 252-271

Stoy R.H., 1968, Second Cape Catalogue for 1950.0, Observations of the sun, moon and planets 1936-1959. Annals of the Cape Observatory, XXIII

Tucker R.H., Buontempo M.E., Gibbs P., Swifte R.H.D., 1983, Third Greenwich Catalogue of Stars, Sun and Moon for 1950.0. Royal Greenwich Observatory Bulletins, No. 187 (data provided on magnetic tape)

Vityazev V.V, 1994, The Rotor: A New Method to Derive Rotation Between Two Reference Frames, Astron. Astrophys. Trans. 4, 195-218 